\title{
A Compact Supermassive Binary Black Hole System
}

\author{
C. Rodriguez ${ }^{1,2}$, G. B. Taylor ${ }^{1,3}$, R. T. Zavala ${ }^{4}$, A. B. Peck ${ }^{5}$, L. K. Pollack ${ }^{6}$ \& R. W. \\ Romani $^{7}$
}

\begin{abstract}
We report on the discovery of a supermassive binary black hole system in the radio galaxy $0402+379$, with a projected separation between the two black holes of just $7.3 \mathrm{pc}$. This is the closest black hole pair yet found by more than two orders of magnitude. These results are based upon recent multi-frequency observations using the Very Long Baseline Array (VLBA) which reveal two compact, variable, flat-spectrum, active nuclei within the elliptical host galaxy of 0402+379. Multiepoch observations from the VLBA also provide constraints on the total mass and dynamics of the system. Low spectral resolution spectroscopy using the Hobby-Eberly Telescope indicates two velocity systems with a combined mass of the two black holes of $\sim 1.5 \times 10^{8} \mathrm{M}_{\odot}$. The two nuclei appear stationary while the jets emanating from the weaker of the two nuclei appear to move out and terminate in bright hot spots. The discovery of this system has implications for the number of close binary black holes that might be sources of gravitational radiation. Green Bank Telescope observations at $22 \mathrm{GHz}$ to search for water masers in this interesting system are also presented.
\end{abstract}

Subject headings: galaxies: active - galaxies: individual $(0402+379)$ - radio continuum: galaxies - radio lines: galaxies

\footnotetext{
${ }^{1}$ Department of Physics and Astronomy, University of New Mexico, Albuquerque, NM 87131

${ }^{2}$ Department of Physics, Universidad Simon Bolivar, Sartenejas, Venezuela

${ }^{3}$ National Radio Astronomy Observatory, Socorro NM 87801

${ }^{4}$ United States Naval Observatory, Flagstaff Station 10391 W. Naval Observatory Rd. Flagstaff, AZ 86001

${ }^{5}$ Harvard-Smithsonian CfA, SMA Project, 645 N. A'ohoku Pl, Hilo, HI 96721

${ }^{6}$ Department of Astronomy \& Astrophysics, University of California at Santa Cruz, Santa Cruz, CA 95064

${ }^{7}$ Department of Physics, Stanford University, Stanford, CA 94305-4060
} 


\section{Report Documentation Page}

Form Approved

OMB No. 0704-0188

Public reporting burden for the collection of information is estimated to average 1 hour per response, including the time for reviewing instructions, searching existing data sources, gathering and maintaining the data needed, and completing and reviewing the collection of information. Send comments regarding this burden estimate or any other aspect of this collection of information,

including suggestions for reducing this burden, to Washington Headquarters Services, Directorate for Information Operations and Reports, 1215 Jefferson Davis Highway, Suite 1204, Arlington

VA 22202-4302. Respondents should be aware that notwithstanding any other provision of law, no person shall be subject to a penalty for failing to comply with a collection of information if it

does not display a currently valid OMB control number.

\begin{tabular}{|c|c|}
\hline $\begin{array}{l}\text { 1. REPORT DATE } \\
\mathbf{0 3} \text { APR } 2006\end{array}$ & $\begin{array}{l}\text { 3. DATES COVERED } \\
\text { - }\end{array}$ \\
\hline \multirow{3}{*}{$\begin{array}{l}\text { 4. TITLE AND SUBTITLE } \\
\text { A Compact Supermassive Binary Black Hole System }\end{array}$} & 5a. CONTRACT NUMBER \\
\hline & 5b. GRANT NUMBER \\
\hline & 5c. PROGRAM ELEMENT NUMBER \\
\hline \multirow[t]{3}{*}{ 6. AUTHOR(S) } & 5d. PROJECT NUMBER \\
\hline & 5e. TASK NUMBER \\
\hline & 5f. WORK UNIT NUMBER \\
\hline $\begin{array}{l}\text { 7. PERFORMING ORGANIZATION NAME(S) AND ADDRESS(ES) } \\
\text { U.S. Naval Observatory Flagstaff Station } \mathbf{1 0 3 9 1} \text { W. Naval Observatory } \\
\text { Rd. Flagstaff, AZ } \mathbf{8 6 0 0 1}\end{array}$ & $\begin{array}{l}\text { 8. PERFORMING ORGANIZATION } \\
\text { REPORT NUMBER }\end{array}$ \\
\hline \multirow[t]{2}{*}{ 9. SPONSORING/MONITORING AGENCY NAME(S) AND ADDRESS(ES) } & 10. SPONSOR/MONITOR'S ACRONYM(S) \\
\hline & $\begin{array}{l}\text { 11. SPONSOR/MONITOR'S REPORT } \\
\text { NUMBER(S) }\end{array}$ \\
\hline
\end{tabular}

12. DISTRIBUTION/AVAILABILITY STATEMENT

Approved for public release, distribution unlimited

13. SUPPLEMENTARY NOTES

The original document contains color images.

14. ABSTRACT

15. SUBJECT TERMS

16. SECURITY CLASSIFICATION OF:

a. REPORT

unclassified b. ABSTRACT

unclassified
17. LIMITATION OF ABSTRACT

SAR
18. NUMBER

OF PAGES

34 19a. NAME OF RESPONSIBLE PERSON unclassified 


\section{Introduction}

Black holes are a direct consequence of the physics described in Einstein's theory of gravity. There is a great deal of indirect astronomical observational evidence for these exotic objects in two mass ranges: stellar mass black holes, with masses of 4-15 times the mass of our Sun; and supermassive black holes, with masses ranging from $10^{5}$ to $10^{10}$ solar masses. There is also some evidence for intermediate-mass black holes, those with masses of a few hundred to a few thousand solar masses (see Filippenko \& Ho 2003, Gebhardt et al. 2005 ).

Since most nearby galaxies harbor supermassive black holes at their centers (Richstone et al. 1998), the merging of galaxies, an essential part of the galaxy formation process, is thought to be the prevalent method in which supermassive black hole binaries are formed. Accordingly, such systems should be common in galaxies. An understanding of the evolution and formation of these systems is important for an understanding of the evolution and formation of galaxies in general.

The evolution of a binary supermassive black hole involves three stages (Begelman et al. 1980), which are summarized by Merritt \& Milosavljević (2005) as follows: (1) As the galaxies merge, the supermassive black holes sink toward the center of the new galaxy via dynamical friction forming a binary; (2) the binary continues to decay mainly due to the interaction of stars on orbits intersecting the binary, which are then ejected at velocities comparable to the binary's orbital velocity, carrying away energy and angular momentum; (3) finally, if the binary's separation decreases to the point where the emission of gravitational waves becomes efficient at carrying away the last remaining angular momentum, the supermassive black holes coalesce rapidly. There is circumstantial evidence that most binary black holes merge in less than a Hubble time (Komossa 2003a). Therefore, the most massive systems that are able to coalesce in less than a Hubble time will create the loudest gravitational wave events in the universe (Sesana et al. 2004), which might be detectable by a low-frequency gravitational wave experiment such as the Laser Interferometer Space Antenna (LISA).

Our ability to resolve both supermassive black holes in any given binary system depends on the separation between them, and on their distance from Earth. It is believed that the longest timescales in the evolution of a supermassive binary black hole system leading up to coalescence is the stage in which the system is closely bound $(\sim 0.1-10 \mathrm{pc})$, meaning that in most of these systems the black hole pair can only be resolved by VLBI observations (see review by Komossa 2003a detailing observational evidence for supermassive black holes binaries). Some source properties like X-shaped radio galaxies and double-double radio galaxies, helical radio-jets, double-horned emission line profiles, and semi-periodic variations in lightcurves have been taken as indirect evidence for compact binary black holes though other explanations are possible. The BL Lacertae Object OJ 287 is a candidate for harboring 
a supermassive binary black hole, inferred from the characteristics of its optical lightcurve, which shows repeated outbursts at 11.86 y intervals (Sillanpää et al. 1988). Combining optical as well as radio observations, Valtaoja et al. (2000) presented a new interpretation which suggests that at intervals of $11.86 \mathrm{y}$, the secondary black hole crosses the accretion disk of the primary black hole, causing a thermal flare visible only in the optical. About a year later, the disturbance propagates down the relativistic jet and results in the growth of new synchroton-emitting shocks visible both in the optical and radio. The observed 11.86 y period corresponds to the orbital period of the compact binary black hole. Some wider systems have, however, been found more directly. The ultra luminous galaxy NGC 6240, discovered by the Chandra X-ray observatory, was found to have a pair of active supermassive black holes at its center (Komossa et al. 2003b), separated by a distance of $1.4 \mathrm{kpc}$. Another system that has been known for some time is the double AGN (7 kpc separation) constituting the radio source $3 \mathrm{C} 75$, which was discovered by the VLA to have two pairs of radio jets (Owen et al. 1985).

In this paper we present further observations of the radio galaxy $0402+379$, which was discovered by Maness et al. (2004) to contain two central, compact, flat spectrum, variable components (designated $\mathrm{C} 1$ and $\mathrm{C} 2$ ), a feature which has not been observed in any other compact source. Maness et al. (2004) remarked upon the unusual properties found in this source and proposed several physical explanations. One possible scenario is for one component to be a foreground or background source, instead of being associated with $0402+379$. However, because of the small separation between C1 and C2 (7.3 pc), and because of a faint bridge of radio emission found connecting components $\mathrm{C} 1$ and $\mathrm{C} 2$, this theory was ruled out. A second explanation was that the nucleus was being gravitationally lensed. However, based on the significant difference in the lightcurves of components $\mathrm{C} 1$ and $\mathrm{C} 2$, and the close proximity of $0402+379(z=0.055)$, this theory was also eliminated. Two other scenarios were proposed by Maness et al. (2004), which could not be conclusively ruled out and remained as possible explanations. The first of these suggest that component C2 could be a knot in the southern jet, with $\mathrm{C} 1$ classified as the core. To test this hypothesis we performed high frequency, high resolution Very Long Baseline Array (VLBA ${ }^{1}$ ) observations of $0402+379$, designed to resolve any jet component and to look for relative motions. The final explanation is that $\mathrm{C} 1$ and $\mathrm{C} 2$ are two active nuclei of a supermassive binary black hole system.

Throughout this discussion, we assume $\mathrm{H}_{0}=75 \mathrm{~km} \mathrm{~s}^{-1} \mathrm{Mpc}^{-1}, \mathrm{q}_{0}=0.5$, and 1 mas $=$ $1.06 \mathrm{pc}$

\footnotetext{
${ }^{1}$ The National Radio Astronomy Observatory is operated by Associated Universities, Inc., under cooperative agreement with the National Science Foundation.
} 


\section{Observations}

\subsection{VLBA Observations from 2005}

VLBA observations were made on 2005 January 24 and June 13 at 0.317, 4.976, 8.410, 15.354, 22.222, and 43.206 GHz. Four IFs with a bandwidth of $8 \mathrm{MHz}$ were observed in 32 channels in both $\mathrm{R}$ and $\mathrm{L}$ circular polarizations. Four-level quantization was employed at all six frequencies. The net integration time on $0402+379$ was 115 minutes at $0.3 \mathrm{GHz}$, 69 minutes at $5 \mathrm{GHz}, 69$ minutes at $8 \mathrm{GHz}, 122$ minutes at $15 \mathrm{GHz}, 251$ minutes at 22 $\mathrm{GHz}$, and 249 minutes at $43 \mathrm{GHz}$. Standard flagging, amplitude calibration, fringe fitting, bandpass calibration (using 3C 84 for bandpass calibration and 3C 111 for gain calibration), and frequency averaging procedures were followed in the Astronomical Image Processing System (AIPS; van Moorsel et al. 1996). Opacity corrections were performed for the 22 and $43 \mathrm{GHz}$ data. AIPS reduction scripts described in Ulvestad et al. (2001) were used for a large part of the reduction. All manual editing, imaging, deconvolution, and self-calibration were done using Difmap (Shepherd et al. 1995).

\subsection{Archival Observations}

To further study this source, we obtained fully-calibrated VLBI data taken in 1990 (Xu et al. 1995), in 1996 (VCS; Beasley et al. 2002), in three epochs (1994, 1996, and 1999) of the CJ Proper Motion Survey (Britzen et al. 2003) and in 2003 (Maness et al. 2004). These data were imaged and modeled in Difmap to aid in analysis of motions, variability, and spectra of $0402+379$. Further information regarding these observations can be found in Table 1.

\subsection{Green Bank Telescope constraints on $\mathrm{H}_{2} \mathrm{O}$ Masers}

Observations were made with the GBT on 2005 October 14 . We used the $18-22 \mathrm{GHz}$ K-band receiver, which uses dual beams separated by $3^{\prime}$ in azimuth. The GBT beamwidth is $\sim 36^{\prime \prime}$ at $22 \mathrm{GHz}$, and pointing uncertainties were $\sim 5^{\prime \prime}$. Pointing was corrected hourly using $0402+379$ itself, which has sufficient continuum emission. The telescope was nodded between two positions on the sky such that one beam was always centered on the position of 0402+379 during integration. The spectrometer was configured with two bandpasses of 200 $\mathrm{MHz}$ each, overlapped by $20 \mathrm{MHz}$ at the redshifted $\mathrm{H}_{2} \mathrm{O}$ frequency of $21.075 \mathrm{GHz}$, so that the total coverage was $\pm 2700 \mathrm{~km} \mathrm{~s}^{-1}$ with respect to the systemic velocity of the galaxy. 
The spectral resolution was equivalent to $0.33 \mathrm{~km} \mathrm{~s}^{-1}$. The zenith system temperature was between 40 and $55 \mathrm{~K}$ for the duration of the run. Atmospheric opacity at $22 \mathrm{GHz}$ was estimated from system temperature and weather data, and ranged from 0.09 to 0.12 at the zenith. The data were reduced using GBTIDL. We found that the higher frequency IF was subject to a $60 \mathrm{MHz}$ ripple across the baseband, most likely the result of known imperfections and temperature sensitivities of the IF transmission. To reduce the effects of the bandpass ripple we subtracted polynomials of order 4 and 8 from the first and second IFs respectively. We were left with some small residual ripples, but the period of $60 \mathrm{MHz}$ is large enough that any maser emission present would have been apparent nonetheless. We Hanning-smoothed the spectra following calibration. The $1 \sigma \mathrm{rms}$ sensitivity of these observations is $\sim 2 \mathrm{mJy}$ per $\mathrm{km} \mathrm{s}^{-1}$. No maser emission was detected.

\subsection{HET Spectroscopy}

We obtained a spectrum of the core of $0402+379$ on 2004 December 11 with the $9.2 \mathrm{~m}$ Hobby-Eberly telescope (HET; Ramsey et al 1998) Marcario Low Resolution Spectrograph (LRS; Hill et al. 1998). Two 600s exposures were taken, using the G3 VPH Grism, a Schott OG 515 blocking filter and a $1.5^{\prime \prime}$ slit placed at the parallactic angle. The resulting spectrum covers $\lambda \lambda=6300-9120 \AA$ at $5.6 \stackrel{\circ}{A}$ resolution. We applied standard IRAF calibrations and find that the spectrum is similar to that obtained by Stickel et al. (1993), with a reddened continuum and Seyfert 2 emission lines at a redshift of $z=0.05523(1)\left(16,460 \mathrm{~km} \mathrm{~s}^{-1}\right)$.

In Figure 1 we show the $\mathrm{H} \alpha$ region of the spectrum, where the only strong lines are present. The lines are resolved with Gaussian width $12.5 \pm 1 \stackrel{\AA}{A}$, after deconvolution of the instrumental resolution. Here the uncertainty is the range in the fitted linewidths for the various species; this substantially exceeds the statistical error. The lines appear asymmetric with a red shoulder suggesting two components with a $\sim 7 \pm 1 \AA$ separation, i.e. a component

velocity separation of $\sim 300 \mathrm{~km} \mathrm{~s}^{-1}$. The residual to the line fit in Figure 1 also shows significant excesses in the line wings, suggesting the presence of a broader $\mathrm{H} \alpha$ component.

\subsection{The Host Galaxy}

Optical imaging is at present quite limited, but in the Palomar Sky survey images $0402+379$ appears as a relatively bright $\mathrm{r}=17.2$ elliptical galaxy embedded in a halo of patchy faint emission, extending to an apparent companion 25" to the NE. Line emission (OI \& NII) is seen from this region in the HET spectra, suggesting the presence of disturbed 
photo-ionized gas. An optical image in Stickel et al. (1993) also shows this faint emission. These authors comment that the elliptical core has a "flat brightness distribution". The 2MASS (Cutri et al. 2003) $\mathrm{J}-\mathrm{K}$ color of $0402+379$ is 1.757 , and this is consistent with the value expected for quasars at 0402+379's redshift (Barkhouse \& Hall 2001) The J-K color could indicate star formation activity from a recent merger, or an obscured AGN consistent with the radio galaxy identification for this source. Additional optical imaging would be useful to understand the dynamical state of $0402+379$ 's core and the origin of the excitation of the surrounding nebulosity.

A source appears near $0402+379$ in the ROSAT Bright source catalog (Voges et al. 1999). 1RXSJ040547.3+380308 gives $0.21 \mathrm{PSPC} \mathrm{cnts} \mathrm{s}^{-1}$. Using the Galactic absorption in this direction $N_{H} \approx 3 \times 10^{21} \mathrm{~cm}^{-2}$, and assuming a typical AGN power law index of $\Gamma=1.7$ this corresponds to an unabsorbed $0.1-2 \mathrm{keV}$ flux of $8.5 \times 10^{-12} \mathrm{erg} \mathrm{cm}^{-2} \mathrm{~s}^{-1}$ or a luminosity $L_{0.1-2} \approx 5 \times 10^{43} \mathrm{erg} \mathrm{s}^{-1}$, i.e. $\sim 2 \times 10^{-26} \mathrm{erg} \mathrm{cm}^{-2} \mathrm{~Hz}^{-1}$. The radio flux density of $0402+379$ at $5 \mathrm{GHz}$ is $1.1 \mathrm{Jy}$ (Pauliny-Toth et al. 1978; Becker et al. 1991) which is a radio luminosity

of $7 \times 10^{31} \mathrm{ergs} \mathrm{sec}^{-1} \mathrm{~Hz}^{-1}$. This is in agreement with the X-ray to $5 \mathrm{GHz}$ radio luminosity correlation of Brinkmann et al. (2000).

The ROSAT archive also contains two HRI pointings of this source for a combined exposure of $27 \mathrm{ks}$. These show that the X-ray source is largely resolved, extending over a radius of $\sim 15^{\prime \prime}$ and appears to follow the faint diffuse emission surrounding the galaxy core. The bulk of the X-ray flux lies between the elliptical and its companion, further supporting the interaction hypothesis. The X-ray luminosity for this diffuse emission is comparable to the AGN estimate above, e.g. $L_{0.1-2} \approx 3 \times 10^{43} \mathrm{erg} \mathrm{s}^{-1}$ for a $1 \mathrm{keV}$ Raymond-Smith plasma, and likely dominates the flux of the ROSAT All-Sky Survey source.

We find that the X-ray emission of $0402+379$ is unique; of the 35 known CSO's (Compact Symmetric Objects) this is the only source detected in the ROSAT All-Sky Survey. Thus, further X-ray observations could abet optical data in probing the nature of this emission and the connection with recent merger and/or nuclear activity.

\section{Results}

\subsection{Radio Continuum}

Figure 2 shows naturally weighted 0.3 and $5 \mathrm{GHz}$ images from the 2005 VLBA observations. The structure of the source at $5 \mathrm{GHz}$ reveals the presence of two diametrically opposed jets, as well as two central strong components, one directly between the jets and the other one also between the jets but offset from the center. Following the convention 
established by Maness et al. (2004), we designated the aligned central component as C2, and the offset central component as $\mathrm{C} 1$. As we can see in Figure 2, the $5 \mathrm{GHz}$ image spans $\sim 40$ mas $(\sim 40 \mathrm{pc})$, corresponding to a small region in the central part of the $0.3 \mathrm{GHz}$ image, which shows structure on scales of $\sim 500$ mas $(\sim 500 \mathrm{pc})$. The orientation of the 0.3 $\mathrm{GHz}$ image is consistent with that seen by the VLA at $5 \mathrm{GHz}$ (Maness et al. 2004). The VLA image shows extended emission going northwards, whereas the northern jet seen in our $5 \mathrm{GHz}$ image is pointing in the northeast direction, suggesting that at some point the jet changes direction and starts moving northwards, probably as a consequence of interactions with the surrounding medium. The VLA 1.5 GHz image (Maness et al. 2004) also shows the extended emission going northwards. The southern jet seen in our $5 \mathrm{GHz}$ image is pointing in the southwest direction, which is consistent with both the $1.5 \mathrm{GHz}$ and the $5 \mathrm{GHz}$ VLA images.

Figure 3 shows naturally weighted $8,15,22$, and $43 \mathrm{GHz}$ images from the 2005 VLBA observations. For both the 8 and $15 \mathrm{GHz}$ images, the overall structure of the source is similar to that at $5 \mathrm{GHz}$, both jets are present as well as the two central components ( $\mathrm{C} 1$ and $\mathrm{C} 2)$.

It is clear that for higher frequencies the two central components are easily distinguished and remain unresolved, while both jets become fainter and are heavily resolved. At $22 \mathrm{GHz}$ these effects are readily apparent, and become more prominent at $43 \mathrm{GHz}$, where the jets can barely be detected. Before these observations were made, the highest frequency for which data had been taken for this source was $15 \mathrm{GHz}$.

Elliptical or circular Gaussian components were fitted to the visibility data using Difmap. We obtained an estimate for the sizes of $\mathrm{C} 1$ and $\mathrm{C} 2$ based on our 15, 22, and $43 \mathrm{GHz}$ model fits. In this case, we fit circular Gaussian components for both $\mathrm{C} 1$ and $\mathrm{C} 2$, obtaining an average value of $0.173 \pm 0.045$ mas or $0.183 \pm 0.048 \mathrm{pc}$ for $\mathrm{C} 1$, and $0.117 \pm 0.033$ mas or $0.124 \pm 0.035 \mathrm{pc}$ for $\mathrm{C} 2$.

\subsection{Component Motions and Variability}

In order to explore questions pertaining to motion and variability in $0402+379$, we obtained fully calibrated $5 \mathrm{GHz}$ VLBI data taken in 1990 (Xu et al. 1995), as well as $5 \mathrm{GHz}$ VLBA data taken in three epochs (1994, 1996, and 1999) of the CJ Proper Motion Survey (Britzen et al. 2003) and in 2003 (Maness et al. 2004). Combining these data with our 2005 observations at $5 \mathrm{GHz}$, we were able to probe motion and variability in this source over a time baseline of $15 \mathrm{y}$.

Motion and variability studies were performed by fitting eight elliptical Gaussian com- 
ponents in Difmap to the 2003 visibility data. Then, we used this model to fit the $5 \mathrm{GHz}$ data corresponding to the 1990, 1994, 1996, 1999, and 2005 epochs. We let only position and flux density vary; all other parameters were held fixed at the 2003 values. Results from our fits are listed in Table 2, and Figure 4 shows the components model, where we have labeled each of them and we also added arrows showing the direction of motion of each component, which will be explained below.

To study component variability in $0402+379$, we compared the flux density for components $\mathrm{C} 1$ and $\mathrm{C} 2$, the mean flux density of the southern components (S1, S2, S3, and S4), and the mean flux density of the northern components (N1 and N2) over each of our 6 epochs at $5 \mathrm{GHz}$. The above regions were chosen primarily on the basis of their isolation relative to other components in the source. Errors for each region were computed on the basis of the rms noise and our estimated absolute flux calibration errors $(\sim 20 \%$ for the 1990 and $1994 \mathrm{Mk}$ II VLBI epochs, and $\sim 5 \%$ for the 1996, 1999, 2003, and 2005 VLBA epochs). The resulting fractional variation light curves are shown in Figure 5. These light curves were created by dividing each region's flux density at each epoch by the mean region flux density found from averaging all epochs. To aid in readability of our graph, the aligned component (C2), the northern lobe, and the southern lobe are displaced on the $y$-axis by 1,2 , and 3 units, respectively.

From Figure 5 and Table 2 we find that component C1 substantially increases in flux over the 15 y baseline, starting from 18 mJy in 1990 and increasing in brightness to 59 $\mathrm{mJy}$ in 2005. We also find that component $\mathrm{C} 2$ is variable, ranging from less than $10 \mathrm{mJy}$ in 1990, to $24 \mathrm{mJy}$ in 1996, and $20 \mathrm{mJy}$ in 2005. Because our 1990 epoch was observed with Mk II VLBI, this apparent variability could be in part attributed to poor data quality. However, the measured flux for all other components in 1990 is quite consistent with that in our later epochs, suggesting that the calculated upper limit for component C2's flux in 1990 is reliable and that the observed variability in this component is significant. For the southern and northern components, we find that there is no substantial variation in the fluxes over the 15 y baseline.

Based on the time variability observed in both $\mathrm{C} 1$ and $\mathrm{C} 2$ we can estimate the size of these components. However, in this case the variability time scale found for $\mathrm{C} 1$ and $\mathrm{C} 2$ is quite long (roughly 5 to $10 \mathrm{y}$ ), which gives a weak upper limit on the sizes of the components of a few parsecs, consistent with the estimate made in $\S 3.1$.

To calculate the relative velocity of the components, we chose component $\mathrm{C} 1$ as the reference, based on its strength, compactness, and isolation relative to other components in the source. We compared the relative motion for each epoch by fitting a line to each component's relative position, split into $x$ and $y$ components, as a function of time. Results 
of this fitting process are listed in Table 3, and plotted in Figure 6.

The results of this analysis reveal significant motion for the northern hot spots N1 and $\mathrm{N} 2$, yielding a value of $0.054 \pm 0.008 \mathrm{mas} / \mathrm{y}$, or $(0.185 \pm 0.008) \mathrm{c}$, and $0.033 \pm 0.006 \mathrm{mas} / \mathrm{y}$, or $(0.114 \pm 0.019)$ c respectively. These results show that the northern jet is moving away from the two central components to the northeast.

For the southern components S2 and S3, significant motion was also found, yielding $0.0073 \pm 0.0025 \mathrm{mas} / \mathrm{y}$, or $(0.0251 \pm 0.0085) \mathrm{c}$, and $0.016 \pm 0.003 \mathrm{mas} / \mathrm{y}$, or $(0.056 \pm 0.010) \mathrm{c}$ respectively. For the other two southern components, S1 and S4, even though the values found for the velocities were larger than those found for S2 and S3, the values obtained in the fitting for $\chi^{2}$ were large, a fact that can be verified by looking at Figures $6(\mathrm{~d})$ and $6(\mathrm{~g})$. However, for the projected $x$ position of component $\mathrm{S} 4$ with time, the $\chi^{2}$ obtained was nearly unity, which gives us some confidence in the motion of this component, at least in the $x$ direction. We conclude then that, on average, the southern jet is moving away from the two central components to the southwest, though more slowly than the northern jet.

The results obtained for $\mathrm{C} 2$ show no significant motion. The value obtained for the limit on the motion of this component is equal to $0.0067 \pm 0.0094$ mas $/ y$, or less than $0.088 \mathrm{c}$.

In Figure 4 we draw arrows showing the direction of motion found for each component, as well as their relative magnitude. It is important to note that we placed arrows even for those components for which we do not claim significant motion.

\subsection{Radio Continuum Spectra}

By appropriately tapering our $22 \mathrm{GHz} 2005$ data, we obtained an image resolution matched to our $8 \mathrm{GHz}$ continuum image. These two images were then combined to generate an image of the spectral index distribution across the source (Figure 7). In both hotspots of the source, N2 and S2, a steep spectrum was found, whereas in both central components, the spectrum is flat. A plot of flux density as a function of frequency is included in the image of the spectral index distribution for both $\mathrm{C} 1$ and $\mathrm{C} 2$. The values for the flux densities used in order to make these plots were measured from matching resolution images. Details regarding these results are listed in Table 4. 


\section{Discussion}

Four possible scenarios were proposed by Maness et al. (2004) in order to explain the unusual properties found in $0402+379$. Two of them were ruled out, but the possibility that $\mathrm{C} 1$ or $\mathrm{C} 2$ was an unusual jet component in a dense ISM could not be conclusively eliminated.

Our high resolution observations confirm the compactness of component $\mathrm{C} 2$, and measure a size of $0.124 \pm 0.035 \mathrm{pc}$. $\mathrm{C} 2$ is found to have no significant motion, whereas significant flux density variability is found. The spectral peak is shown to be at $\sim 10 \mathrm{GHz}$. It is possible that either or both $\mathrm{C} 1$ and/or $\mathrm{C} 2$ could be a jet component lit up in a collision with a dense interstellar medium. In this scenario the low observed velocity $(<0.088 \mathrm{c})$ is due to the impact with the ISM, and the spectrum is modified by local acceleration of particles. Difficulties with the jet component explanation are (1) it requires a dramatic change in the jet axis on timescales of a few 10s of years, while the larger scale emission (see Figure 2) indicates that the jet axis has been fairly stable on time scales up to $10^{4} \mathrm{y}$; (2) a large gradient in density needs to be invoked to decelerate $\mathrm{C} 1$ and/or $\mathrm{C} 2$ but allow the hotspots to advance (Maness et al. 2004); (3) if C2 is the core responsible for the observed jets and hot spots, and $\mathrm{C} 1$ is a jet component, then the counterjet is conspicuous in its absence given the orientation of the source close to the plane of the sky indicated by the source symmetry.

The absence of a jet associated with $\mathrm{C} 1$ might at first seem unusual since only 6 of $87(7 \%)$ sources in the First Caltech-Jodrell Bank VLBI survey (CJ1 - the survey in which $0402+379$ was imaged by Xu et al. 1995 ) show naked cores with no sign of a jet at $5 \mathrm{GHz}$. At lower flux levels, however, which are more appropriate since $\mathrm{C} 1$ by itself would not have made it into the CJ1 sample, the fraction of naked cores increases to 6 of 24 sources $(25 \%$ Taylor et al. 2005 ). Thus the absence of a jet from $\mathrm{C} 1$ is not by itself evidence against the identification of $\mathrm{C} 1$ as an AGN.

The characteristics found in $\mathrm{C} 1$ and $\mathrm{C} 2$ are typical of AGN; in a complete survey of 32 sources imaged at $43 \mathrm{GHz}$, (Lister 2001) found no unresolved, isolated jet components. This, together with the morphology of $0402+379$, leads us to surmise that neither $\mathrm{C} 1$ nor $\mathrm{C} 2$ is likely to be a jet component. This leaves the remaining most likely explanation that $\mathrm{C} 1$ and $\mathrm{C} 2$ are both active black holes in a compact system. For the remainder of the discussion we

assume that this is the case and explore what we can learn about such a system from the present observations. 


\subsection{Constraints on the mass of the black holes}

We can use the HET and the VLBA observations to obtain kinematic constraints on the mass of the black hole system. At our observed separation, an orbital velocity of $300 \mathrm{~km} / \mathrm{s}$ implies a system mass of $1.5 \times 10^{8}\left(v / 300 \mathrm{~km} \mathrm{~s}^{-1}\right)^{2}(r / 7.3 \mathrm{pc}) \mathrm{M}_{\odot}$. The suggested line splitting from the optical spectra thus implies a mass of a few times $10^{8} \mathrm{M}_{\odot}$. If both radio nuclei are also optically active and show comparable emission line strengths, as expected, then the observed Gaussian line widths put a limit on the line FWHM of $\sim 1300 \mathrm{~km} \mathrm{~s}^{-1}$, implying a limit on the combined black hole mass of $\sim 3 \times 10^{9} \mathrm{M}_{\odot}$. Note that the Hi absorption profiles of Maness et al. (2004) also found velocity structure of $\sim 1000 \mathrm{~km} \mathrm{~s}^{-1}$ extending over a transverse distance of $\sim 20 \mathrm{pc}$. On this scale the mass contribution from gas and stars is not significant, so the limit obtained reflects the mass of the binary black hole system and indicates a high mass system. The implied mass, $\sim 5 \times 10^{9} \mathrm{M}_{\odot}$, could be dominated by the AGN, if the absorption occurs in the nuclear region. Alternatively the two velocity systems might be probing line-of-sight velocities in the products of a recent merger.

An alternative estimate for the central compact object mass can be derived from the blue luminosity of the host bulge (Kormendy \& Gebhardt 2001). The observed V magnitude of $0402+379$ is $\sim 18.5$ (Wills et al. 1973), after correcting for the Galactic $A_{V} \sim 1.6$ and a median B-V color index of $\sim 0.9$ for 484 ellipticals in the Uppsala General Catalogue (UGC), we obtain a B magnitude of 17.8. This corresponds to a central compact object

mass of $\sim 7 \times 10^{7} \mathrm{M}_{\odot}$, in reasonable accord with the estimates above, given that this may still be a disturbed system.

\subsection{Supermassive Binary Black Hole Orbital Parameters}

Using our system mass estimate equal to $1.5 \times 10^{8} \mathrm{M}_{\odot}$ and the projected radial separation between them derived from our 2005 maps (7.3 pc), we find from Kepler's Laws that the period of rotation for such a binary supermassive black hole system should be $\sim 1.5 \times 10^{5}$ y. This period corresponds to a relative projected velocity between components $\mathrm{C} 1$ and $\mathrm{C} 2$ of $\sim 0.001 \mathrm{c}$. The upper limit found for component $\mathrm{C} 1,<0.088 \mathrm{c}$, is consistent with the expected relative velocity between $\mathrm{C} 1$ and $\mathrm{C} 2$, assuming a stable, Keplerian orbit. To actually constrain the masses of the black holes would require observations over a longer time baseline $(\sim 100 \mathrm{y})$. 


\subsection{Gravitational Wave Signal}

What sort of gravitational wave signal might a binary supermassive black hole in $0402+379$ generate? Assuming the current separation is $7.3 \mathrm{pc}$ and the total mass is $1.5 \times 10^{8} \mathrm{M}_{\odot}$ the natural gravitational wave frequency (Hughes 2003) is approximately

$2 \times 10^{-13} \mathrm{~Hz}$. This is well below the expected minimum frequency of LISA. Although 0402+379 may be a long way from generating a detectable gravitational wave signal it may represent a source of noise important for future observations of cosmologically produced gravitational radiation. Ultra low frequency gravitational radiation generated during inflation (Hughes 2003) has an upper limit of $10^{-13} \mathrm{~Hz}$. Thus, a population of black hole binaries like 0402+379 may generate substantial noise which could interfere with the detection of the physics of inflation. An estimate of this noise contribution requires a population synthesis model (e.g., Sesana et al. 2005) which is beyond the scope of this work.

The final stage in the evolution of a binary black hole system is the gravitational radiation stage, where the semimajor axis decreases to the point at which gravitational radiation becomes the dominant dissipative force. A binary black hole on a circular orbit will merge within the time (Peters 1964):

$$
t_{\text {merge }}(a)=5.8 \times 10^{6}\left(\frac{a}{0.01 \mathrm{pc}}\right)^{4}\left(\frac{10^{8} \mathrm{M}_{\odot}}{m_{1}}\right)^{3} \frac{m_{1}^{2}}{m_{2}\left(m_{1}+m_{2}\right)} \mathrm{y}
$$

where $m_{1}$ and $m_{2}$ are the masses of the black holes, and $a$ is the separation between them, once the system is in the final stage. Using the projected radial separation between the black holes we measure for 0402+379 (7.3 pc) and assuming they both have a mass equal to $\sim 10^{8} \mathrm{M}_{\odot}$, we obtain a merger time equal to $\sim 10^{18} \mathrm{y}$. Some other loss of angular momentum will be necessary if this system is to merge in less than a Hubble time.

It is not clear whether, at present, dynamical friction losses or gas dissipative effects (see Komossa 2003a , Merritt \& Milosavljević 2005 ) are strong enough to bring the binary to the gravitational radiation loss regime within a Hubble time. This relates to the issue of the probability of catching the binary at its present (modest) separation. Are we seeing a recent merger in the act of core coalescence or has the binary stalled and must now await loss-cone replenishment and/or re-supply of nuclear gas? The fact that both nuclei are active (radio bright) suggests on-going accretion and implies dissipation today. Only a larger sample of imaged active nuclei can address the fraction of the population in this state, for example the VLBA Imaging and Polarization Survey (VIPS, Taylor et al. 2005 ) which will image 1169 sources. 


\subsection{Jet Components}

Assuming that $\mathrm{C} 2$ is the origin of the radio emission on parsec scales, we can constrain the orientation of $0402+379$. In the simple beaming model for simultaneously ejected jet components moving in opposite directions, both the arm length ratio $D$ and the flux density ratio $R$ depend on the intrinsic speed $\beta=v / c$ and the angle of the twin jets to the line of sight $\theta$ (Taylor \& Vermeulen 1997). The arm length ratio, $D$, is given by

$$
D=\frac{\mu_{\mathrm{N}}}{\mu_{\mathrm{S}}}=\frac{d_{\mathrm{N}}}{d_{\mathrm{S}}}=\left(\frac{1+\beta \cos \theta}{1-\beta \cos \theta}\right),
$$

where the apparent projected distances from C2 (assumed to be the origin of radio emission) are $d_{\mathrm{N}}$ for the northern jet (approaching side) and $d_{\mathrm{S}}$ for the southern jet (receding side), and the apparent proper motions are $\mu_{\mathrm{N}}$ for the northern jet and $\mu_{\mathrm{S}}$ for the southern jet. Similarly, the flux density ratio, $R$, between the northern and southern jet is

$$
R=\frac{S_{\mathrm{N}}}{S_{\mathrm{S}}}=\left(\frac{1+\beta \cos \theta}{1-\beta \cos \theta}\right)^{k-\alpha}
$$

where $\alpha$ is the spectral index, $k=2$ for a continuous jet, and $k=3$ for discrete jet components (c.f. Lind \& Blandford 1985).

In order to determine the arm length ratio we calculated the distances from N2 and S2 to C2 (using the values shown in Table 2), yielding a value of $D=d_{\mathrm{N}} / d_{\mathrm{S}}=2.42$.

For the proper motion ratio we proceeded as follows. We determined a flux density weighted average velocity, for both the northern and southern components, with respect to $\mathrm{C} 2$, using the values shown in Table 3 (calculating the $x$ and $y$ components separately and then calculating the resultant value). Following this procedure we obtained a value of $\mu_{\mathrm{N}} / \mu_{\mathrm{S}}=6.12$.

The flux density ratio was obtained dividing the sum of the fluxes of the northern components by the sum of the fluxes of the southern components (see Table 2), yielding a value of $S_{\mathrm{N}} / S_{\mathrm{S}}=0.32$.

If Doppler boosting dominates the appearance of $0402+379$ then all the ratios calculated should be in agreement according to Equations 2 and 3. However, using $k=2$ and estimating a value for the spectral index of both the north and south hotspots of $\alpha=-1$ (see Table 4 ), we find that $D=2.42, \mu_{\mathrm{N}} / \mu_{\mathrm{S}}=6.12$, and $R^{\frac{1}{k-\alpha}}=0.68$, results that are far from agreement, thus ruling out Doppler boosting as the dominant effect. A possible explanation 
for this discrepancy could be an enhancement in the density of the interstellar medium to the south of the core, which will reduce the velocity in that direction; slow the expansion over time, thus changing the arm length ratio; and increase the flux density of the southern jet. Additional supporting evidence for this hypothesis comes from the enhancement in HI opacity to the southwest (Maness et al. 2004). Assuming that the density enhancement is a recent phenomenon, we can use the arm length ratio obtained in addition to Equation 2 to get $\beta \cos \theta=0.4$. This result implies that the intrinsic velocity must be at least $0.4 \mathrm{c}$ and $\theta$ must be less than $66^{\circ}$.

Using the relative velocity between the northern and southern jets (calculated from the values obtained for the flux density weighted averaged velocities of both the southern and northern components) as well as the distance between components S2 and N2, we estimated the age of the current radio emission to be $\sim 500 \mathrm{y}$. If the slower jet velocities for the southern jet are used then the age for the southern lobe is three times as large. Note that these jet ages are much less than the $\sim 10^{5}$ y binary period in $\S 4.2$, so we expect no significant orbital displacement of the jet components.

\section{Conclusion}

Based on the compactness, motion, variability, and spectra of the two central components in $0402+379$ we conclude that they are both active nuclei of a single galaxy. This pair of AGN forms the closest binary black hole system yet discovered with a projected separation of $7.3 \mathrm{pc}$. The total mass of the system is estimated to be $1.5 \times 10^{8} \mathrm{M}_{\odot}$, and the gravitational radiation frequency to be $2 \times 10^{-13} \mathrm{~Hz}$. Energy losses due to gravitational radiation are not yet significant, so that other mechanisms must be invoked if the orbit is to decay. $0402+379$ may be the tip of an iceberg for a population of supermassive black hole binaries with parsec scale separations. Such a population may produce significant gravitational wave radiation which may need to be considered for the detection of gravitational radiation in the ultra to very low frequency bands.

Having found one system in the CJF sample (Taylor et al. 1996) of 293 sources, we might expect to find others in a larger survey. The VIPS (Taylor et al. 2005) will image 1169 sources and hopefully find additional compact binary black holes.

Facilities: VLBA, GBT, HET

We thank an anonymous referee for constructive comments. This research has made use of the NASA/IPAC Extragalactic Database (NED) which is operated by the Jet Propulsion 
Laboratory, Caltech, under contract with NASA. The National Radio Astronomy Observatory is a facility of the National Science Foundation operated under a cooperative agreement by Associated Universities, Inc.

\section{REFERENCES}

Barkhouse, W. A., \& Hall, P. B. 2001, AJ, 121, 2843

Beasley, A. J., Gordon, D., Peck, A. B., Petrov, L., MacMillan, D. S., Fomalont, E. B., \& Ma, C. 2002, ApJS, 141, 13

Becker, R. H., White, R. L., \& Edwards, A. L. 1991, ApJS, 75, 1

Begelman, M. C., Blandford, R. D., \& Rees, M. J. 1980, Nature, 287, 307

Brinkmann, W., Laurent-Muehleisen, S. A., Voges, W., Siebert, J., Becker, R. H., Brotherton, M. S., White, R. L., \& Gregg, M. D. 2000, A\&A, 356, 445

Britzen, S., et al. 2003, Future Directions in High Resolution Astronomy: A Celebration of the 10th Anniversary of the VLBA, edited by J. D. Romney and M. J. Reid. Socorro, N.M. : National Radio Astronomy Observatory, 2003., p.85, 85

Cutri, R. et al. 2003, 2MASS Extended Source Catalog, NASA/IPAC Extragalactic Database

Filippenko, A. V., \& Ho, L. C. 2003, ApJ, 588, L13

Gebhardt, K., Rich, R. M., \& Ho, L. C. 2005, ApJ, 634, 1093

Hill, G. J., Nicklas, H. E., MacQueen, P. J., Tejada, C., Cobos Duenas, F. J., \& Mitsch, W. 1998, Proc. SPIE, 3355, 375

Hughes, S. A. 2003, Annals of Physics, 303, 142

Komossa, S. 2003, AIP Conf. Proc. 686: The Astrophysics of Gravitational Wave Sources, 686,161

Komossa, S., Burwitz, V., Hasinger, G., Predehl, P., Kaastra, J. S., \& Ikebe, Y. 2003, ApJ, 582, L15

Kormendy, J., \& Gebhardt, K. 2001, AIP Conf. Proc. 586: 20th Texas Symposium on relativistic astrophysics, 586, 363

Lister, M. L. 2001, ApJ, 562, 208 
Lind, K. R., \& Blandford, R. D. 1985, ApJ, 295, 358

Maness, H. L., Taylor, G. B., Zavala, R. T., Peck, A. B., \& Pollack, L. K. 2004, ApJ, 602, 123

Merritt, D., \& Milosavljević, M. 2005, Living Reviews in Relativity, 8, 8

Owen, F. N., Odea, C. P., Inoue, M., \& Eilek, J. A. 1985, ApJ, 294, L85

Pauliny-Toth, I. I. K., Witzel, A., Preuss, E., Kühr, H., Fomalont, E. B., Davis, M. M., \& Kellermann, K. I. 1978, AJ, 83, 451

Peters, P. C. 1964, Phys. Rev. B, 136, 1224

Ramsey, L. W., et al. 1998, Proc. SPIE, 3352, 34

Richstone, D., et al. 1998, Nature, 395, A14

Sesana, A., Haardt, F., Madau, P., \& Volonteri, M. 2004, ApJ, 611, 623

Sesana, A., Haardt, F., Madau, P., \& Volonteri, M. 2005, ApJ, 623, 23

Shepherd, M. C., Pearson, T.J., \& Taylor, G.B. 1995, BASS, 27, 903

Sillanpää, A., Haarala, S., Valtonen, M. J., Sundelius, B., \& Byrd, G. G. 1988, ApJ, 325, 628

Stickel, M., Kuehr, H., \& Fried, J. W. 1993, A\&AS, 97, 483

Taylor, G. B., Readhead, A. C. S., \& Pearson, T. J. 1996, ApJ, 463, 95

Taylor, G. B., \& Vermeulen, R. C. 1997, ApJ, 485, L9

Taylor, G. B., et al. 2005, ApJS, 159, 27

Ulvestad, J., Greisen, E. W. \& Mioduszewski, A. 2001, AIPS Memo 105:AIPS Procedures for initial VLBA Data Reduction, NRAO

Valtaoja, E., Teräsranta, H., Tornikoski, M., Sillanpää, A., Aller, M. F., Aller, H. D., \& Hughes, P. A. 2000, ApJ, 531, 744

van Moorsel, G., Kemball, A., \& Greisen, E. 1996, ASP Conf. Ser. 101: Astronomical Data Analysis Software and Systems V, 5, 37

Voges, W., et al. 1999, A\&A, 349, 389 
Wills, B. J., Wills, D., \& Douglas, J. N. 1973, AJ, 78, 521

Xu, W., Readhead, A. C. S., Pearson, T. J., Polatidis, A. G., \& Wilkinson, P. N. 1995, ApJS, 99, 297 
Table 1. Observations

\begin{tabular}{lccccccccc}
\hline \hline $\begin{array}{c}\text { Frequency } \\
(\mathrm{GHz})\end{array}$ & Instrument & Date & $\begin{array}{c}\text { Time } \\
(\mathrm{min})\end{array}$ & $\begin{array}{c}\text { BW } \\
(\mathrm{MHz})\end{array}$ & Pol. & IFs & $\begin{array}{c}\text { Peak } \\
(\mathrm{Jy} / \text { beam })\end{array}$ & $\begin{array}{c}\text { rms } \\
(\mathrm{mJy})\end{array}$ & Reference \\
\hline 0.32 & VLBA & 2005 Jun 13 & 115 & 8 & 2 & 4 & 0.32 & 0.59 & This paper \\
4.99 & VLBI Mk 2 & 1990 Mar 10 & 80 & 2 & 1 & 1 & 0.17 & 0.94 & Xu et al. 1995 \\
4.99 & VLBI Mk 2 & 1994 Sep 17 & 57 & 2 & 1 & 1 & 0.17 & 1.6 & Britzen et al. 2003 \\
4.99 & VLBI & 1996 Aug 19 & 41 & 8 & 1 & 1 & 0.16 & 0.63 & Britzen et al. 2003 \\
4.99 & VLBA & 1999 Nov 26 & 35 & 8 & 2 & 2 & 0.18 & 0.42 & Britzen et al. 2003 \\
5.00 & VLBA & 2003 Mar 02 & 478 & 16 & 2 & 1 & 0.16 & 0.21 & Maness et al. 2003 \\
4.98 & VLBA & 2005 Jan 24 & 69 & 8 & 2 & 4 & 0.13 & 0.12 & This paper \\
8.41 & VLBA & 2005 Jun 13 & 69 & 8 & 2 & 4 & 0.06 & 0.06 & This paper \\
15.35 & VLBA & 2005 Jan 24 & 122 & 8 & 2 & 4 & 0.05 & 0.17 & This paper \\
22.22 & VLBA & 2005 Jun 13 & 251 & 8 & 2 & 4 & 0.03 & 0.22 & This paper \\
43.21 & VLBA & 2005 Jan 24 & 249 & 8 & 2 & 4 & 0.02 & 0.25 & This paper \\
\hline
\end{tabular}


Table 2. Gaussian Model Components* .

\begin{tabular}{|c|c|c|c|c|c|c|c|c|}
\hline Component & Epoch & $\begin{array}{c}S \\
(\mathrm{Jy})\end{array}$ & $\begin{array}{c}r \\
(\mathrm{mas})\end{array}$ & $\begin{array}{c}\theta \\
\left(^{o}\right)\end{array}$ & $\begin{array}{c}a \\
\text { (mas) }\end{array}$ & $b / a$ & $\begin{array}{c}\Phi \\
\left({ }^{o}\right)\end{array}$ & $\chi^{2}$ \\
\hline \multirow[t]{6}{*}{$\mathrm{C} 1 \ldots$} & 1990.185 & $0.018 \pm 0.004$ & 0.0 & 0.0 & 0.525 & 1.00 & 172.4 & 1.13 \\
\hline & 1994.708 & $0.040 \pm 0.009$ & 0.0 & 0.0 & 0.525 & 1.00 & 172.4 & 0.90 \\
\hline & 1996.630 & $0.040 \pm 0.003$ & 0.0 & 0.0 & 0.525 & 1.00 & 172.4 & 1.31 \\
\hline & 1999.899 & $0.050 \pm 0.004$ & 0.0 & 0.0 & 0.525 & 1.00 & 172.4 & 1.28 \\
\hline & 2003.162 & $0.060 \pm 0.004$ & 0.0 & 0.0 & 0.525 & 1.00 & 172.4 & 2.07 \\
\hline & 2005.062 & $0.059 \pm 0.003$ & 0.0 & 0.0 & 0.525 & 1.00 & 172.4 & 1.67 \\
\hline \multirow[t]{6}{*}{$\mathrm{C} 2 \ldots$} & 1990.185 & $<0.010$ & & & & & & \\
\hline & 1994.708 & $0.025 \pm 0.006$ & 7.323 & 286.21 & 1.61 & 1.00 & 207.9 & 0.90 \\
\hline & 1996.630 & $0.024 \pm 0.002$ & 6.932 & 284.58 & 1.61 & 1.00 & 207.9 & 1.31 \\
\hline & 1999.899 & $0.018 \pm 0.001$ & 6.803 & 282.00 & 1.61 & 1.00 & 207.9 & 1.28 \\
\hline & 2003.162 & $0.021 \pm 0.001$ & 6.809 & 282.72 & 1.61 & 1.00 & 207.9 & 2.07 \\
\hline & 2005.062 & $0.020 \pm 0.001$ & 6.876 & 283.40 & 1.61 & 1.00 & 207.9 & 1.67 \\
\hline \multirow[t]{6}{*}{$\mathrm{S} 1 \ldots$} & 1990.185 & $0.078 \pm 0.017$ & 11.063 & 254.74 & 3.29 & 1.00 & 308.3 & 1.13 \\
\hline & 1994.708 & $0.090 \pm 0.020$ & 12.154 & 251.22 & 3.29 & 1.00 & 308.3 & 0.90 \\
\hline & 1996.630 & $0.087 \pm 0.006$ & 11.978 & 251.46 & 3.29 & 1.00 & 308.3 & 1.31 \\
\hline & 1999.899 & $0.090 \pm 0.006$ & 11.600 & 251.83 & 3.29 & 1.00 & 308.3 & 1.28 \\
\hline & 2003.162 & $0.119 \pm 0.007$ & 11.846 & 251.19 & 3.29 & 1.00 & 308.3 & 2.07 \\
\hline & 2005.062 & $0.103 \pm 0.005$ & 11.876 & 252.10 & 3.29 & 1.00 & 308.3 & 1.67 \\
\hline \multirow[t]{6}{*}{$\mathrm{S} 2 \ldots$} & 1990.185 & $0.291 \pm 0.064$ & 14.119 & 248.45 & 1.51 & 1.00 & 302.3 & 1.13 \\
\hline & 1994.708 & $0.228 \pm 0.050$ & 14.364 & 248.46 & 1.51 & 1.00 & 302.3 & 0.90 \\
\hline & 1996.630 & $0.211 \pm 0.015$ & 14.234 & 248.71 & 1.51 & 1.00 & 302.3 & 1.31 \\
\hline & 1999.899 & $0.198 \pm 0.014$ & 14.120 & 248.66 & 1.51 & 1.00 & 302.3 & 1.28 \\
\hline & 2003.162 & $0.208 \pm 0.012$ & 14.165 & 248.88 & 1.51 & 1.00 & 302.3 & 2.07 \\
\hline & 2005.062 & $0.159 \pm 0.008$ & 14.192 & 248.84 & 1.51 & 1.00 & 302.3 & 1.67 \\
\hline \multirow[t]{6}{*}{ S3.. } & 1990.185 & $0.187 \pm 0.041$ & 16.029 & 253.40 & 3.40 & 0.37 & 32.0 & 1.13 \\
\hline & 1994.708 & $0.168 \pm 0.037$ & 16.144 & 252.75 & 3.40 & 0.37 & 32.0 & 0.90 \\
\hline & 1996.630 & $0.158 \pm 0.011$ & 16.149 & 252.48 & 3.40 & 0.37 & 32.0 & 1.31 \\
\hline & 1999.899 & $0.152 \pm 0.011$ & 16.084 & 252.39 & 3.40 & 0.37 & 32.0 & 1.28 \\
\hline & 2003.162 & $0.186 \pm 0.011$ & 16.015 & 252.48 & 3.40 & 0.37 & 32.0 & 2.07 \\
\hline & 2005.062 & $0.153 \pm 0.008$ & 15.980 & 252.48 & 3.40 & 0.37 & 32.0 & 1.67 \\
\hline \multirow[t]{6}{*}{$\mathrm{S} 4 \ldots$} & 1990.185 & $0.006 \pm 0.001$ & 17.454 & 251.27 & 2.72 & 0.10 & 333.8 & 1.13 \\
\hline & 1994.708 & $0.028 \pm 0.006$ & 17.399 & 252.60 & 2.72 & 0.10 & 333.8 & 0.90 \\
\hline & 1996.630 & $0.019 \pm 0.001$ & 17.731 & 250.16 & 2.72 & 0.10 & 333.8 & 1.31 \\
\hline & 1999.899 & $0.019 \pm 0.001$ & 17.641 & 248.96 & 2.72 & 0.10 & 333.8 & 1.28 \\
\hline & 2003.162 & $0.029 \pm 0.002$ & 17.763 & 249.71 & 2.72 & 0.10 & 333.8 & 2.07 \\
\hline & 2005.062 & $0.027 \pm 0.001$ & 17.861 & 249.79 & 2.72 & 0.10 & 333.8 & 1.67 \\
\hline \multirow[t]{6}{*}{$\mathrm{N} 1 \ldots$} & 1990.185 & $0.107 \pm 0.024$ & 17.033 & 5.15 & 13.58 & 0.27 & 20.3 & 1.13 \\
\hline & 1994.708 & $0.093 \pm 0.020$ & 18.678 & 4.72 & 13.58 & 0.27 & 20.3 & 0.90 \\
\hline & 1996.630 & $0.086 \pm 0.006$ & 19.143 & 5.95 & 13.58 & 0.27 & 20.3 & 1.31 \\
\hline & 1999.899 & $0.085 \pm 0.006$ & 18.874 & 5.45 & 13.58 & 0.27 & 20.3 & 1.28 \\
\hline & 2003.162 & $0.105 \pm 0.006$ & 19.271 & 6.13 & 13.58 & 0.27 & 20.3 & 2.07 \\
\hline & 2005.062 & $0.091 \pm 0.005$ & 19.353 & 6.15 & 13.58 & 0.27 & 20.3 & 1.67 \\
\hline \multirow[t]{3}{*}{$\mathrm{N} 2 \ldots$} & 1990.185 & $0.044 \pm 0.010$ & 21.103 & 12.86 & 2.38 & 1.00 & 254.2 & 1.13 \\
\hline & 1994.708 & $0.057 \pm 0.013$ & 21.414 & 11.47 & 2.38 & 1.00 & 254.2 & 0.90 \\
\hline & 1996.630 & $0.049 \pm 0.003$ & 21.416 & 13.17 & 2.38 & 1.00 & 254.2 & 1.31 \\
\hline
\end{tabular}


Table 2-Continued

\begin{tabular}{ccccccccc}
\hline \hline Component & \multirow{2}{*}{ Epoch } & $\begin{array}{c}S \\
(\mathrm{Jy})\end{array}$ & $\begin{array}{c}r \\
(\mathrm{mas})\end{array}$ & $\begin{array}{c}\theta \\
\left({ }^{\circ}\right)\end{array}$ & $\begin{array}{c}a \\
(\mathrm{mas})\end{array}$ & $b / a$ & $\begin{array}{c}\Phi \\
\left({ }^{o}\right)\end{array}$ & $\chi^{2}$ \\
\hline & 1999.899 & $0.056 \pm 0.004$ & 21.487 & 13.15 & 2.38 & 1.00 & 254.2 & 1.28 \\
& 2003.162 & $0.068 \pm 0.004$ & 21.613 & 13.42 & 2.38 & 1.00 & 254.2 & 2.07 \\
& 2005.062 & $0.051 \pm 0.003$ & 21.585 & 13.39 & 2.38 & 1.00 & 254.2 & 1.67 \\
\hline
\end{tabular}

*NOTE - Parameters of each Gaussian component of the model brightness distribution are as follows: Component, Gaussian component; Epoch, year of observation (see Table 1 and $\S 1$ ); $S$, flux density; $r, \theta$, polar coordinates of the center of the component relative to the center of component C1 (Beasley et al. (2002) calculated the position of the source to be $\sim \mathrm{RA}$ $04 \mathrm{~h} 05 \mathrm{~m} 49.2623 \mathrm{~s} \mathrm{Dec}+38^{\circ} 03^{\prime} 32^{\prime \prime} .235$. We are assuming component $\mathrm{C} 1$ to be approximately at this location) ; $a$, semimajor axis; $b / a$, axial ratio; $\Phi$, component orientation; $\chi^{2}$, goodnessof-fit for eight component model in each epoch. All angles are measured from north through east. Errors in flux are based on our absolute amplitude calibration as well as the rms noise. Note that due to the complicated morphology of the source, variability studies were performed using components $\mathrm{C} 1, \mathrm{C} 2$, the sum of the southern components (S1, S2, S3, and S4), and the sum of the northern components (N1 and N2).

Table 3. Component Motion Fitting Results.

\begin{tabular}{lccccccc}
\hline \hline Component & $\begin{array}{c}\text { Velocity in } y \\
\text { (mas/y) }\end{array}$ & $\chi^{2}$ & $\begin{array}{c}\text { Velocity in } x \\
\text { (mas/y) }\end{array}$ & $\chi^{2}$ & $\begin{array}{c}\text { Velocity } \\
(\text { mas/y) }\end{array}$ & $\begin{array}{c}\text { Velocity } \\
(\mathrm{c})\end{array}$ & $\begin{array}{c}\text { Angle of Motion * } \\
\left({ }^{o}\right)\end{array}$ \\
\hline C1 & Reference Component & $\ldots$ & $\ldots$ & $\ldots$ & $\ldots$ & $\ldots$ & $\ldots$ \\
C2 & $-0.006 \pm 0.007$ & 2.02 & $0.003 \pm 0.007$ & 0.60 & $0.007 \pm 0.009$ & $0.023 \pm 0.032$ & 153.4 \\
S1 & $-0.027 \pm 0.003$ & 9.66 & $-0.024 \pm 0.003$ & 6.90 & $0.036 \pm 0.004$ & $0.124 \pm 0.015$ & 221.6 \\
S2 & $0.007 \pm 0.002$ & 0.35 & $-0.002 \pm 0.002$ & 1.34 & $0.007 \pm 0.002$ & $0.025 \pm 0.009$ & 344.5 \\
S3 & $-0.013 \pm 0.002$ & 1.30 & $0.010 \pm 0.002$ & 0.34 & $0.016 \pm 0.003$ & $0.056 \pm 0.010$ & 142.4 \\
S4 & $-0.016 \pm 0.006$ & 5.85 & $-0.026 \pm 0.006$ & 1.04 & $0.031 \pm 0.008$ & $0.105 \pm 0.028$ & 238.4 \\
N1 & $0.046 \pm 0.006$ & 1.90 & $0.028 \pm 0.006$ & 1.30 & $0.054 \pm 0.008$ & $0.185 \pm 0.028$ & 31.3 \\
N2 & $0.023 \pm 0.004$ & 0.68 & $0.024 \pm 0.004$ & 2.59 & $0.033 \pm 0.006$ & $0.114 \pm 0.019$ & 46.2 \\
\hline
\end{tabular}

*Angles measured from north through east. 
Table 4. Continuum Spectrum Results* .

\begin{tabular}{|c|c|c|c|c|c|c|c|}
\hline Component & $\begin{array}{c}\text { Frequency } \\
\quad(\mathrm{GHz})\end{array}$ & $\begin{array}{l}\text { Flux } \\
(\mathrm{mJ})\end{array}$ & $\alpha_{5-8}$ & $\alpha_{8-15}$ & $\alpha_{8-22}$ & $\alpha_{15-22}$ & $\alpha_{22-43}$ \\
\hline \multirow[t]{5}{*}{$\mathrm{C} 1 \ldots$} & 4.987 & $53.2 \pm 2.6$ & \multirow[t]{5}{*}{$0.25 \pm 0.19$} & \multirow[t]{5}{*}{$-0.24 \pm 0.25$} & \multirow[t]{5}{*}{$-0.55 \pm 0.15$} & \multirow[t]{5}{*}{$-1.07 \pm 0.54$} & \multirow{5}{*}{$-0.62 \pm 0.30$} \\
\hline & 8.421 & $60.5 \pm 3.0$ & & & & & \\
\hline & 15.365 & $52.4 \pm 5.2$ & & & & & \\
\hline & 22.233 & $35.3 \pm 3.5$ & & & & & \\
\hline & 43.217 & $23.4 \pm 2.3$ & & & & & \\
\hline \multirow[t]{5}{*}{$\mathrm{C} 2 \ldots$} & 4.987 & $10.7 \pm 0.5$ & \multirow[t]{5}{*}{$0.68 \pm 0.19$} & \multirow[t]{5}{*}{$-0.06 \pm 0.26$} & \multirow[t]{5}{*}{$-0.37 \pm 0.16$} & \multirow[t]{5}{*}{$-0.88 \pm 0.55$} & \multirow[t]{5}{*}{$-0.44 \pm 0.31$} \\
\hline & 8.421 & $15.3 \pm 0.8$ & & & & & \\
\hline & 15.365 & $14.8 \pm 1.5$ & & & & & \\
\hline & 22.233 & $10.7 \pm 1.1$ & & & & & \\
\hline & 43.217 & $8.0 \pm 0.8$ & & & & & \\
\hline \multirow[t]{5}{*}{$\mathrm{S} 2 \ldots$} & 4.987 & $72.2 \pm 3.5$ & \multirow[t]{5}{*}{$-0.42 \pm 0.19$} & \multirow[t]{5}{*}{$-0.73 \pm 0.25$} & \multirow[t]{5}{*}{$-1.11 \pm 0.16$} & \multirow[t]{5}{*}{$-1.73 \pm 0.55$} & \multirow[t]{5}{*}{$-1.24 \pm 0.31$} \\
\hline & 8.421 & $57.8 \pm 3.0$ & & & & & \\
\hline & 15.365 & $37.2 \pm 3.7$ & & & & & \\
\hline & 22.233 & $19.6 \pm 2.0$ & & & & & \\
\hline & 43.217 & $8.6 \pm 0.9$ & & & & & \\
\hline \multirow[t]{5}{*}{$\mathrm{N} 2 \ldots$} & 4.987 & $11.2 \pm 0.5$ & \multirow[t]{5}{*}{$-0.18 \pm 0.18$} & \multirow[t]{5}{*}{$-0.56 \pm 0.24$} & \multirow[t]{5}{*}{$-0.89 \pm 0.15$} & \multirow[t]{5}{*}{$-1.43 \pm 0.51$} & \multirow[t]{5}{*}{$\ldots$} \\
\hline & 8.421 & $10.2 \pm 0.5$ & & & & & \\
\hline & 15.365 & $7.3 \pm 0.7$ & & & & & \\
\hline & 22.233 & $4.3 \pm 0.4$ & & & & & \\
\hline & 43.217 & undetected & & & & & \\
\hline
\end{tabular}

${ }^{*}$ Results obtained from the 2005 VLBA observations. The fluxes were measured from matching resolution images. See $\S 3.3$ for more details. 


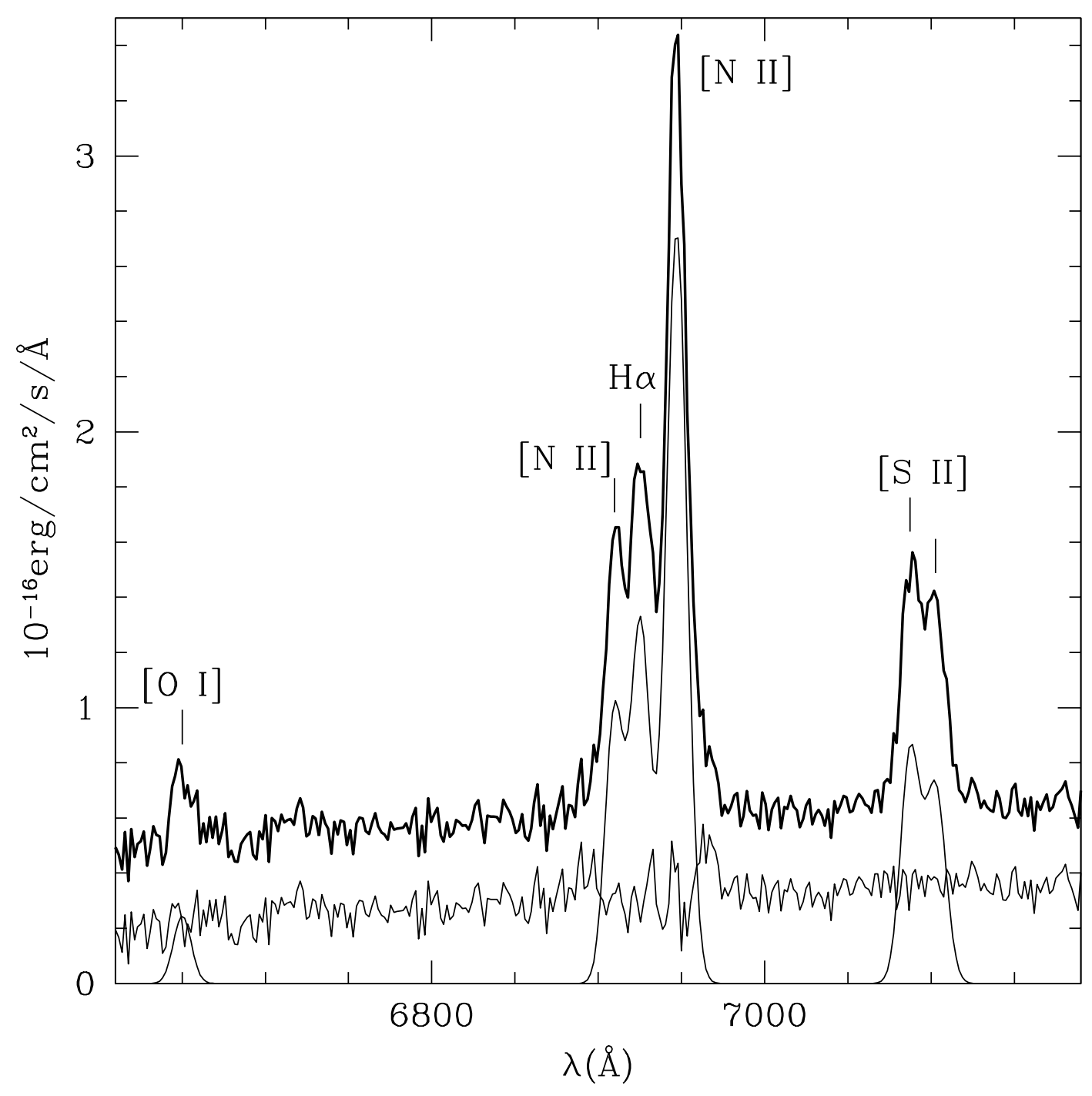

Fig. 1. - The optical spectrum at 5.6 $\AA$ spectral resolution taken by the Hobby-Eberly Telescope. The thick line is the spectrum, the thin line shows the single component model and the residual spectrum. 


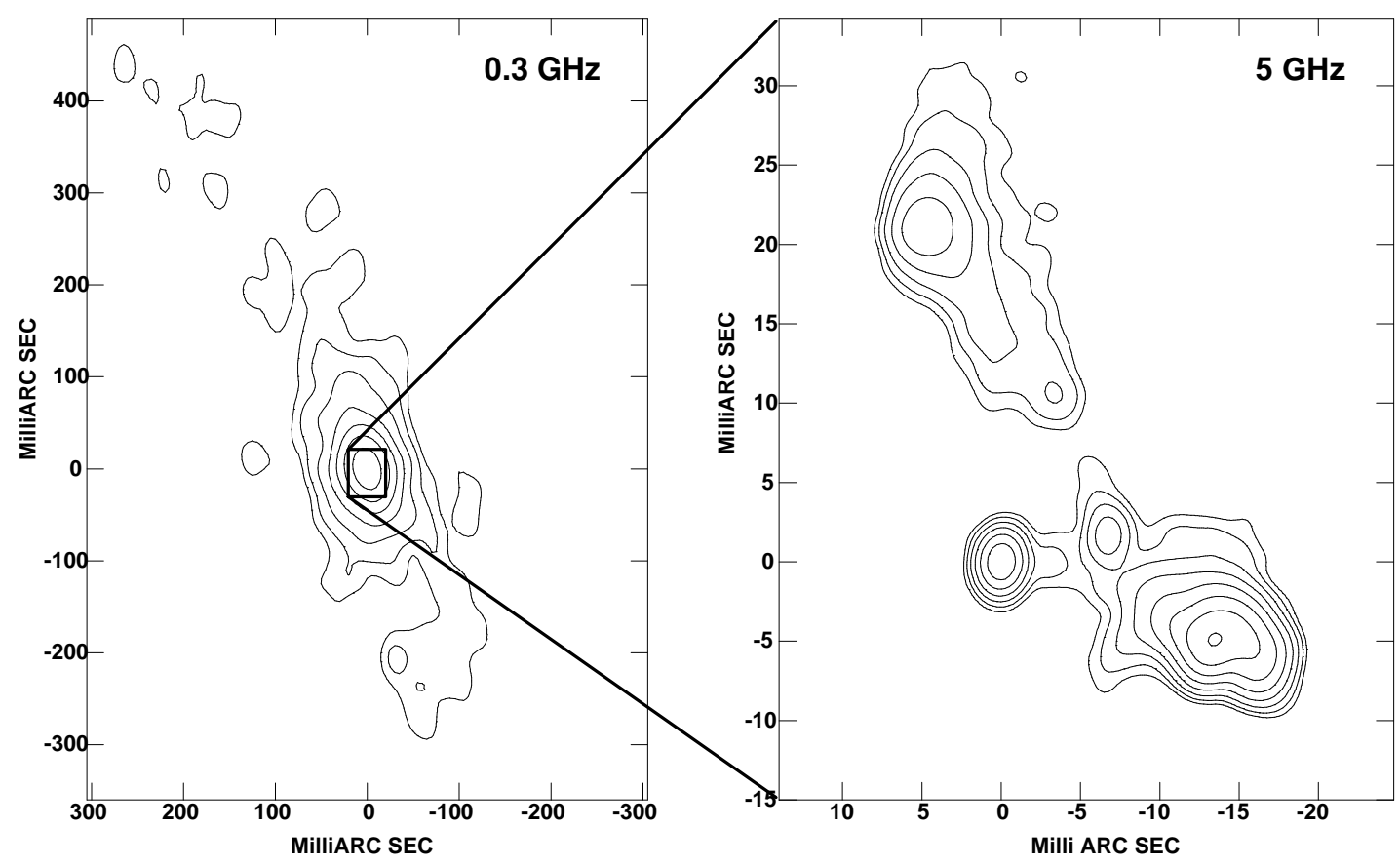

Fig. 2.- Naturally weighted 2005 VLBA images of $0402+379$ at 0.3 and $5 \mathrm{GHz}$. Contours are drawn beginning at $3 \sigma$ and increase by factors of 2 thereafter. The peak flux density and rms noise for each frequency are given in Table 1. Right ascension and declination are relative to $\mathrm{C} 1$, as shown in Figure 3, assumed to be at $\sim \mathrm{RA} 04 \mathrm{~h} 05 \mathrm{~m} 49.2623 \mathrm{~s}$ Dec $+38^{\circ} 03^{\prime} 32^{\prime \prime} .235$ (Beasley et al. 2002). 

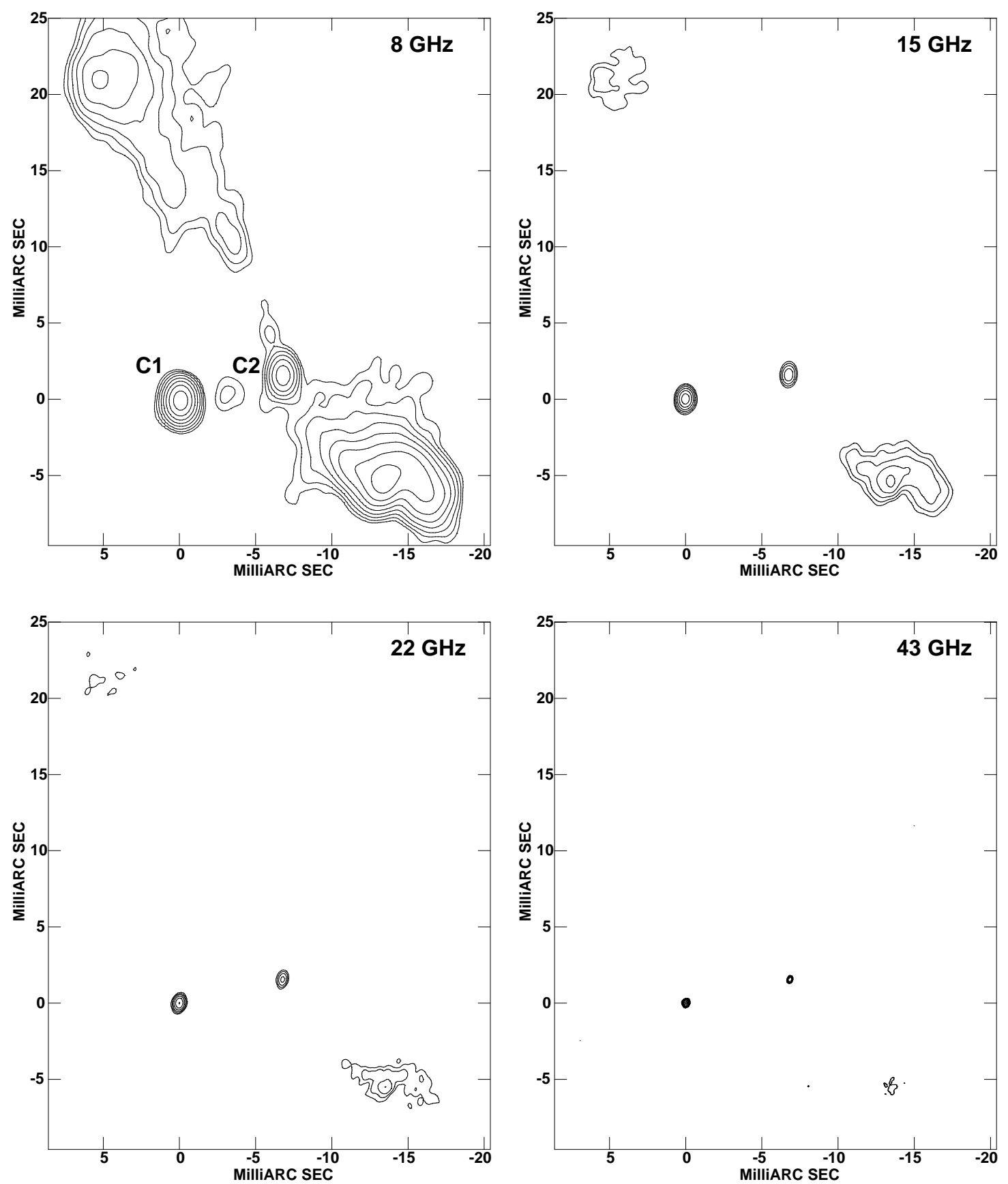

Fig. 3.- Naturally weighted 2005 VLBA images of $0402+379$ at 8, 15, 22 and $43 \mathrm{GHz}$. Contours are drawn beginning at $3 \sigma$ and increase by factors of 2 thereafter. The peak flux density and rms noise for each frequency are given in Table 1. The labels shown in the $5 \mathrm{GHz}$ map indicate the positions of the two strong, compact, central components derived from model-fitting. 


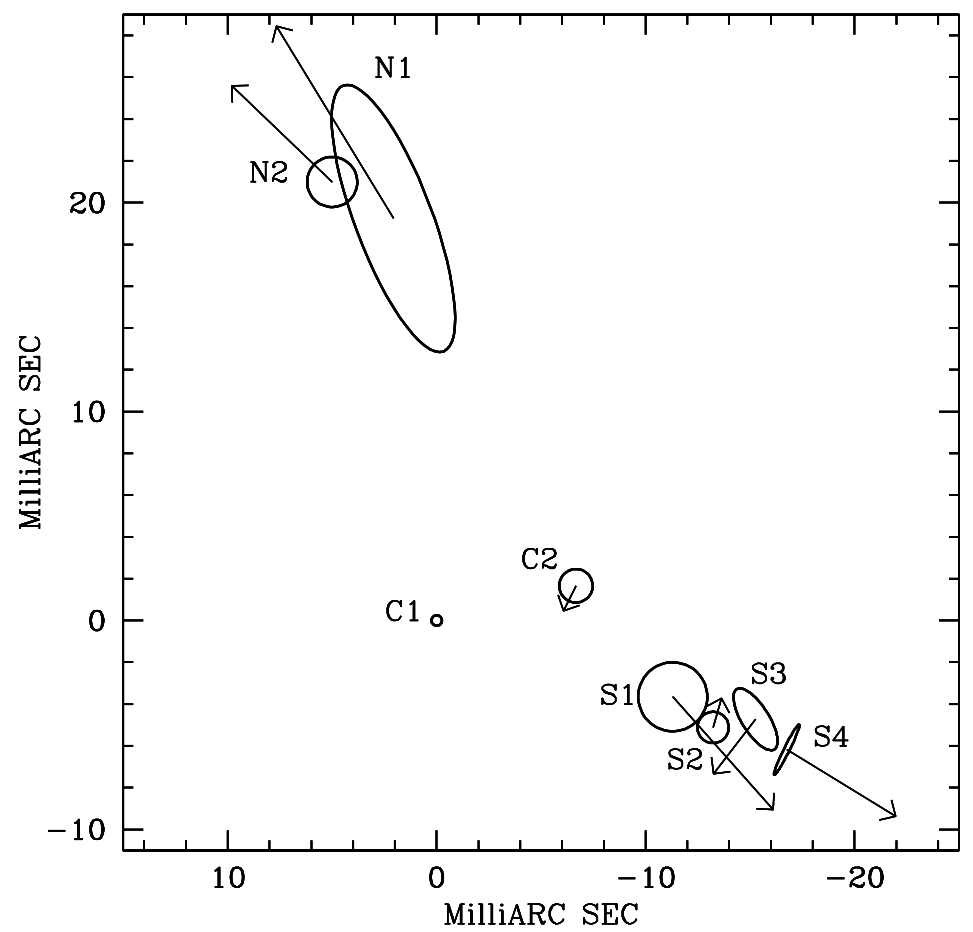

Fig. 4.- Components model for the VLBA observations of $0402+379$ at frequencies of $5 \mathrm{GHz}$ and above. The arrows shown represent the direction of motion found for each component, relative to the position of $\mathrm{C} 1$, obtained from a time baseline of $15 \mathrm{y}$. Arrow lengths have been multiplied by a factor of 200 . See $\S 3.2$ for more details. Note that we placed arrows even for those components for which we are not claiming motion. The specific model shown corresponds to a frequency of $5 \mathrm{GHz}$. 


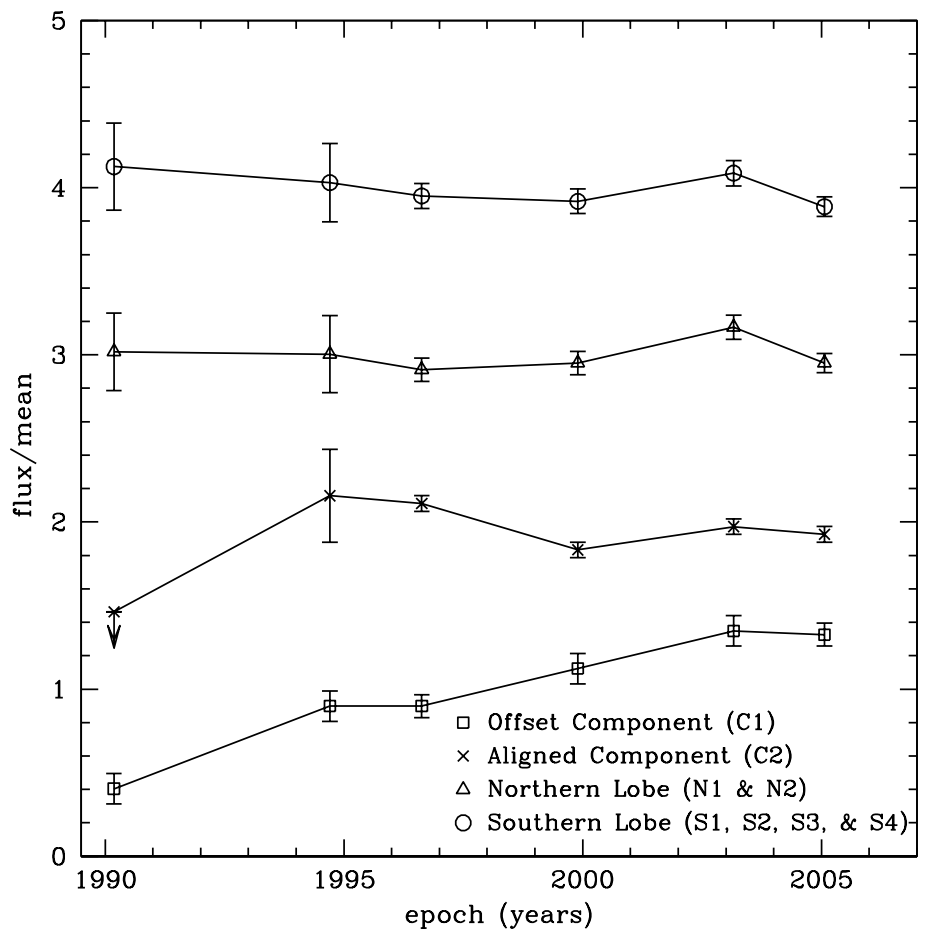

Fig. 5.- Light curves of the different components of $0402+379$ at $5 \mathrm{GHz}$. The flux densities that produced this graph were taken from Table 2 and are discussed in $§ 3.2$. The displayed light curves were created by dividing each region's flux at each epoch by the mean region flux found from averaging all observed epochs. The aligned core candidate, the northern lobe, and the southern lobe are displayed on the $y$-axis by 1, 2, and 3 units, respectively. Errors are estimated from the rms noise and the absolute flux calibration errors for each epoch. 
(a)
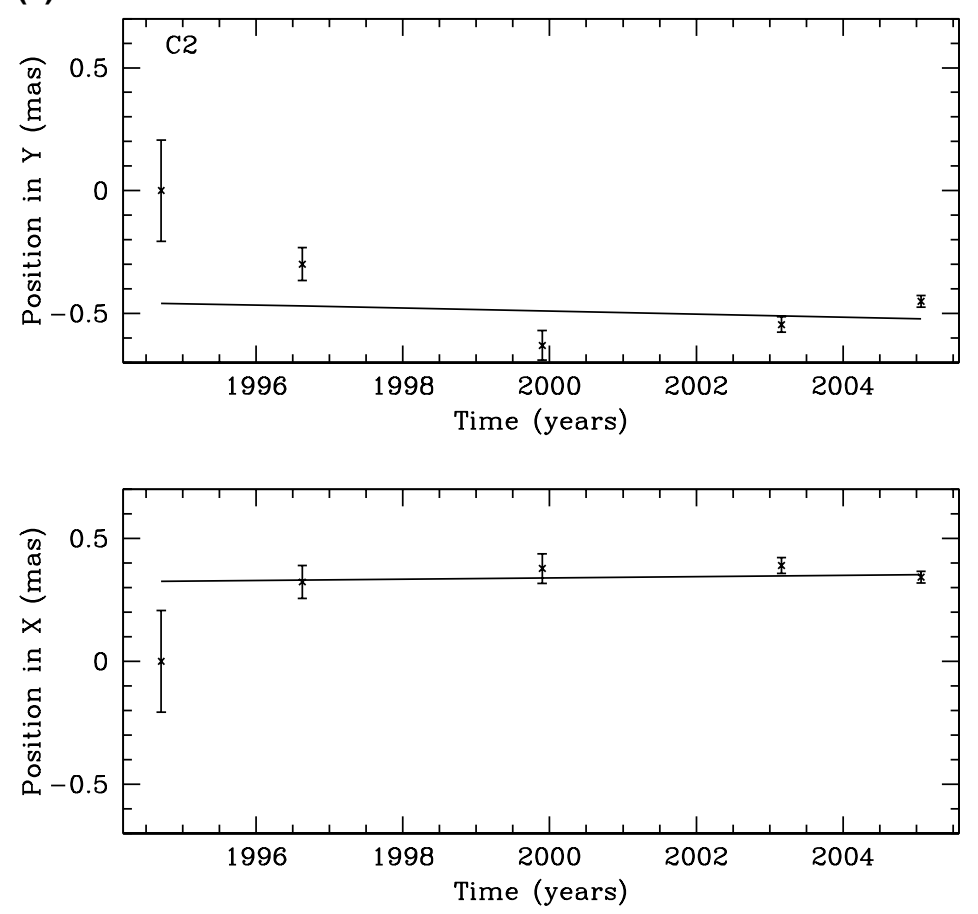

Fig. 6.- (a) Projected $x$ and $y$ positions of component C2 with time. The $x$ and $y$ components of velocity are shown as a solid line. (b) Same for component N1. (c) Same for component N2. (d) Same for component S1. (e) Same plot component S2. (f) Same component S3. (g) Same for component S4. See Table 3 for more details. 
(b)
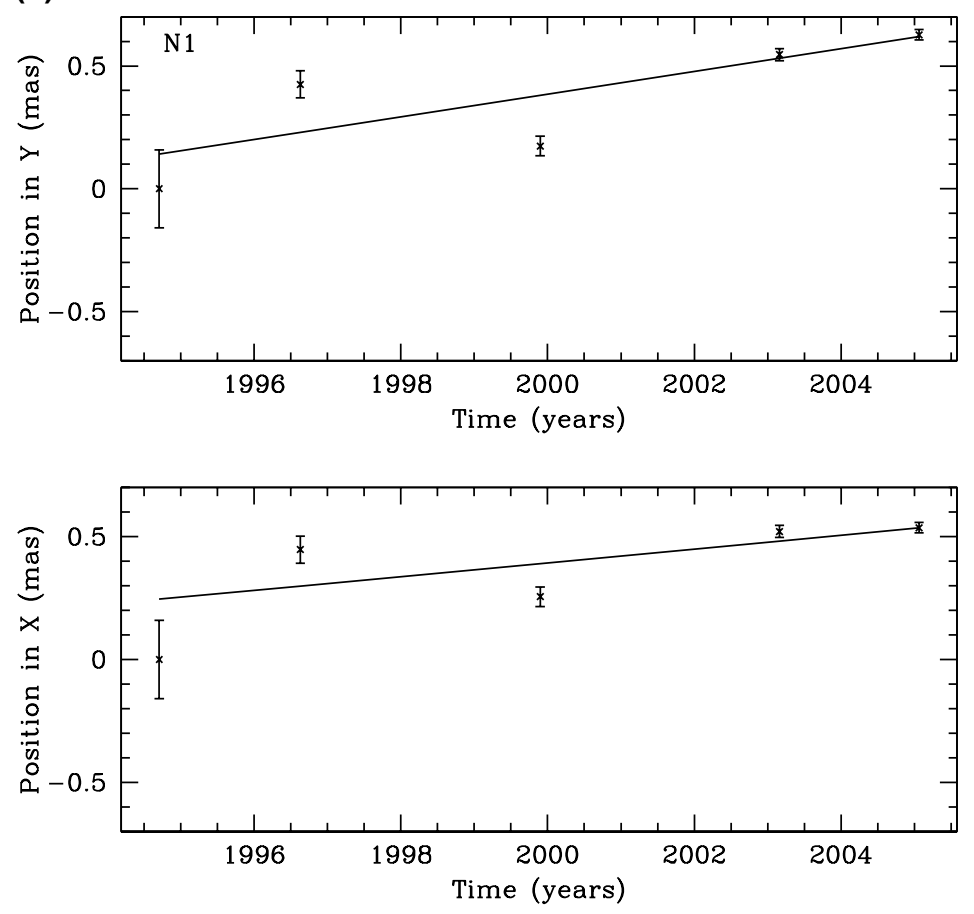

Fig. 6.- Figure 6 continued 
(c)
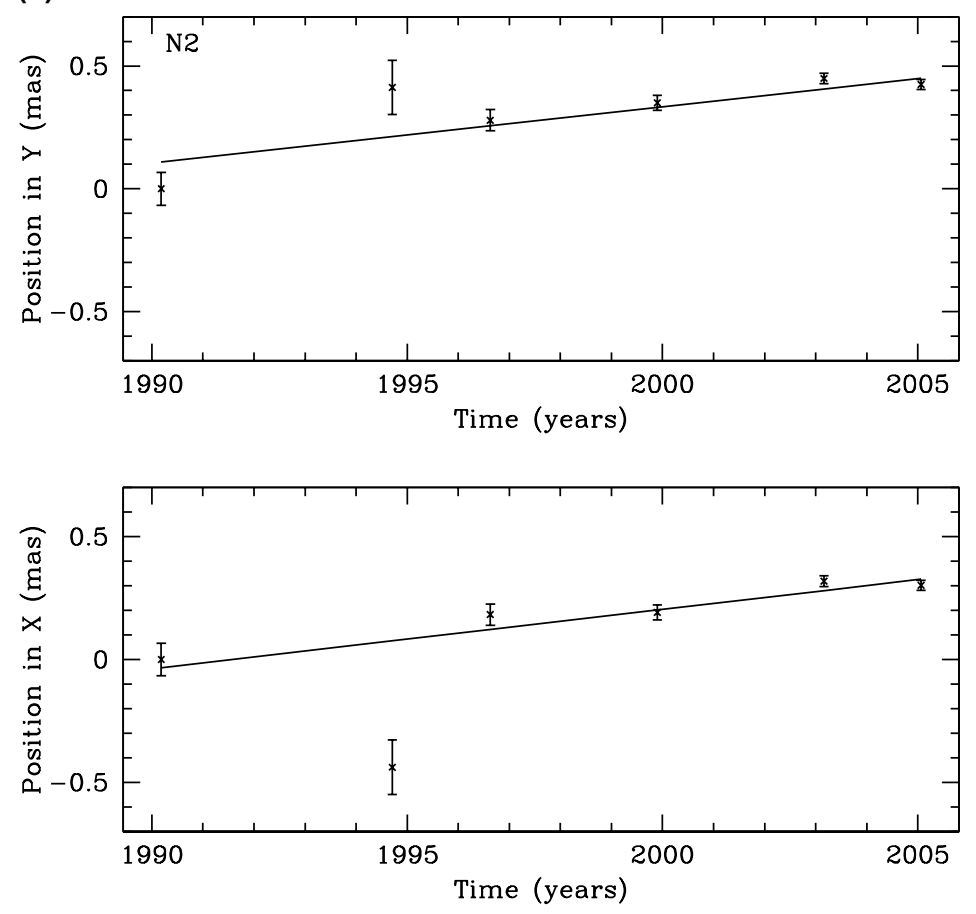

Fig. 6.- Figure 6 continued 
(d)


Fig. 6.- Figure 6 continued 
(e)


Fig. 6.- Figure 6 continued 
(f)
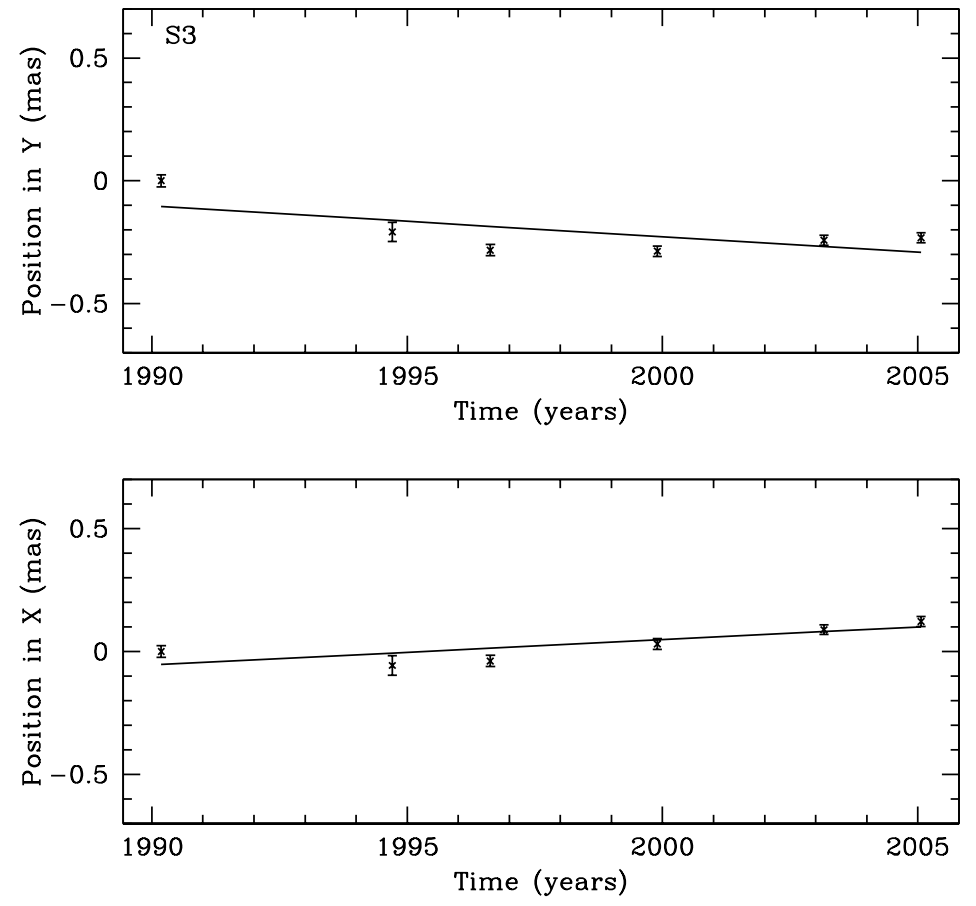

Fig. 6.- Figure 6 continued 
(g)
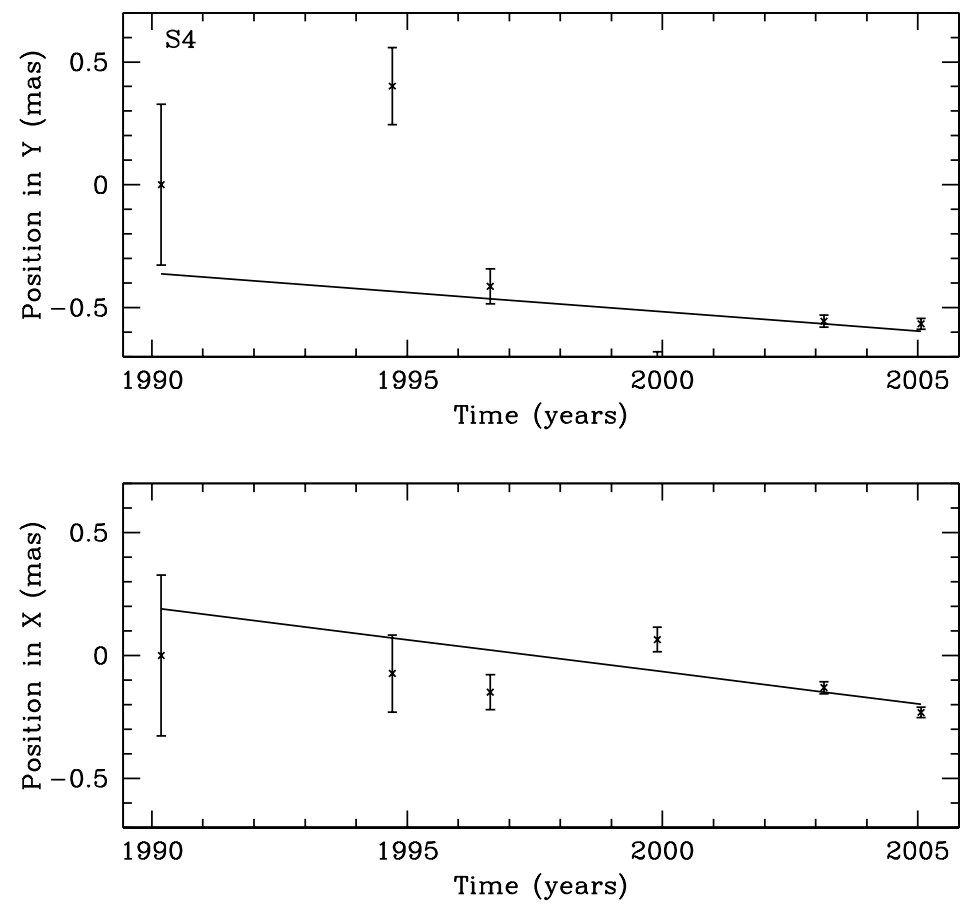

Fig. 6.- Figure 6 continued 


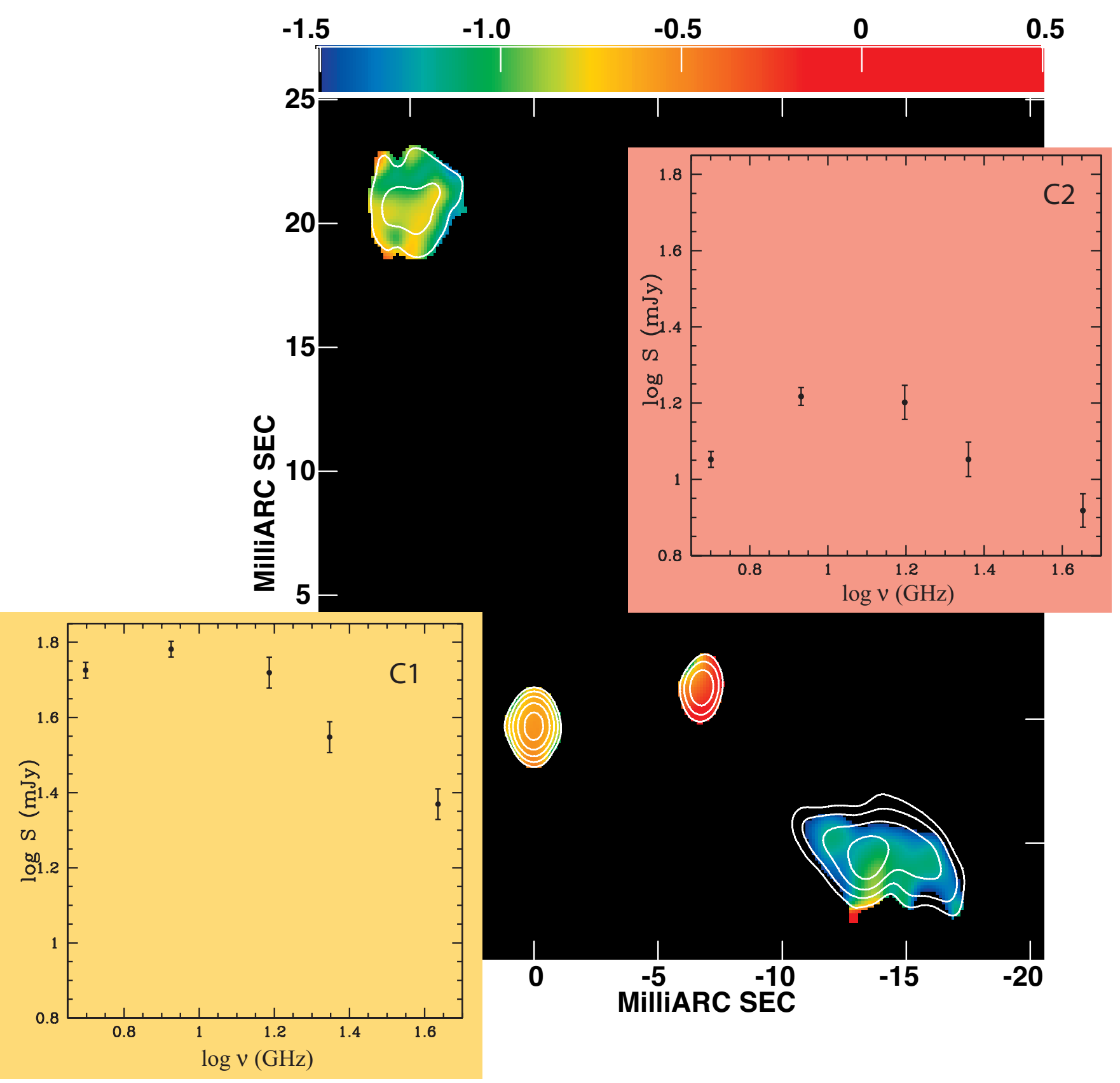

Fig. 7.- Spectral index distribution between 8 and $22 \mathrm{GHz}$ from the 2005 VLBA observations. The contours are taken from the $22 \mathrm{GHz}$ observations and are set at $7 \sigma$, increasing by a factor of 2 thereafter. 